\title{
NUMERICAL PREDICTION OF THE EFFECTS OF MINIATURE CHANNEL SHAPED SCRATCHES ON THE FRACTURE BEHAVIOUR OF WIRES FOR CIVIL ENGINEERING APPLICATIONS USING FINITE ELEMENT ANALYSIS
}

\begin{abstract}
K.K. ADEWOLE ${ }^{1}$ AND S.J. BULL ${ }^{2}$
The effects of the miniature channel-shaped scratches not detectable by the present inline electromagnetic defect detection system employed for wires' surface defect detection on the fracture behaviour of the wires for civil engineering applications were investigated numerically. Finite element analysis revealed that both miniature channel-shaped across-the-thickness and acrossthe-width scratches change the fracture behaviour of the wires in terms of the fracture initiation locations and fracture process sequence. However, miniature across-the-thickness scratches does not affect the fracture shape of the wire while miniature across-the-width scratches changed the wires' cup and cone fracture to a fracture shape with a predominantly flat fracture. These results provide an understanding of the fracture behaviour of wires with miniature scratches and serve as an alternative or a complimentary tools to experimental or fractographic failure analysis of wires with miniatures scratches which are difficult to carry out in the laboratory due to the sizes of the scratches.
\end{abstract}

Keywords: Wires, Failure analysis, Finite element, Fracture behaviour, Scratches

\section{INTRODUCTION}

The understanding of how carbon steel wires used for civil engineering applications deform and fracture is a necessary first step towards addressing the issue of the fracture performance the wires, which as stated by [1] remains a major concern in civil engineering construction and maintenance of wire-reinforced structures such as pre-stressed concrete structures. The understanding of the fracture process starting from the identification of the fracture origin(s), the determination of the fracture sequence, and the identification of any macroscopic features/defects or microstructural abnormalities that are responsible and/or contribute to fracture initiation or propagation is also necessary for failure analysis and serves as an important tool for quality control [2]. Consequently, the

1 School of Chemical Engineering and Advanced Materials, Newcastle University, Newcastle upon Tyne, United Kingdom, NE1 7RU, E-mail: kkadewole@yahoo.com

2 School of Chemical Engineering and Advanced Materials, Newcastle University, Newcastle upon Tyne, United Kingdom, NE1 7RU, E-mail: s.j.bull@ncl.ac.uk 
understanding of the fracture process of wires used for civil engineering applications is necessary for failure analysis and would serves as an important tool for the quality control of the wires. Furthermore, the understanding of the fracture behavior of wires with surface defects such as scratches, cracks etc is particularly necessary because the wires, as a typical engineering material, inevitably contain defects which could affect their fracture behaviour. The presence of surface scratches on aircraft target towing cables made up of carbon steel wire wires similar to the carbon steel wires used for civil engineering applications has been identified by [3] to be instrumental to the failure of the wires. This explains why the manufacturer and the users of wires used for civil engineering applications (such as the flexible pipe manufacturers) desire an understanding of the effects of miniatures surface scratches with depth less than $0.2 \mathrm{~mm}$ that are not detectable by the present inline electromagnetic defect detection system employed for wires' surface defects detection on the fracture behaviour of the wires.

Scratches on the surface of the wires could be caused by the wires previous manufacturing processes such as rolling and/or drawing operations and by mechanical damage to the surface of the wires during transportation, construction and installation [4]. The published work on the failure analysis and fracture performance of wires for civil engineering applications such as the experimental works of [1,5 and 6] shows the influence of notch-like defects (with emphasis on the stress state and triaxiality around the defect) and the degree of cold drawing on the fracture behaviour of pre-stressing steel wires. These authors employed fractographic analysis of the scanning electron microscopy (SEM) images of the fractured wire surfaces to establish the microscopic topographies, micro-fracture maps (MFM) and the micromechanisms of fracture in the wires. While it is possible to conduct failure analyses and understand the fracture behaviour of wires by studying the fractured surfaces of wires obtained from laboratory tests because the fracture initiation point and the entire fracture process(s) can be readily established, it may not be possible to do the same for wires that fracture in service. This is because after a service failure, the fracture features (fracture initiation and propagation marks) could be obliterated or drastically altered due to the destruction of the fracture face(s) by the fracture incident or by the post-fracture events [1]. The obliteration or drastic alteration of the fracture features could make failure analyses and/or the understanding the fracture behaviour of failed components (in this case, the failed wires) difficult, if not impossible [2]. Consequently, there is the need to employ alternatives methods to purely experimental work to conduct failure analyses and for the understanding of the fracture behaviour of wires.

An alternative method to the use of purely experimental work in the investigation of fracture or failure analysis of civil engineering structures recently highlighted by [7] is the simulation-based investigation of the fracture of civil engineering structures. In particular, simulation-based investigation of fracture of civil engineering structures allows for parametric studies which can examine situations which may not be feasible to be investigated experimentally [7]. Simulation-based investigation also provides researchers with a tool to develop insights into localized effects that trigger fracture [7], 
which may be impossible to establish experimentally in situations where the fracture features have been obliterated or drastically altered by the fracture incident or by the post-fracture events as stated by [2].

In this work, the effects of the miniature across-the-thickness and across-the-width channel-shaped scratches with widths and depths below $0.2 \mathrm{~mm}$ on the fracture behaviour of typical carbon steel wires used for civil engineering construction were investigated because the miniature scratches cannot be detected by the inline electromagnetic defect detection system presently in use for detecting defects on the surface of the wires. The scratches considered in this work are channel-shaped which represent the typical shape of the flat bottom scratches that run across the width and thickness of the as-received wires as shown by the environmental scanning electron micrographs shown in Figure 1.
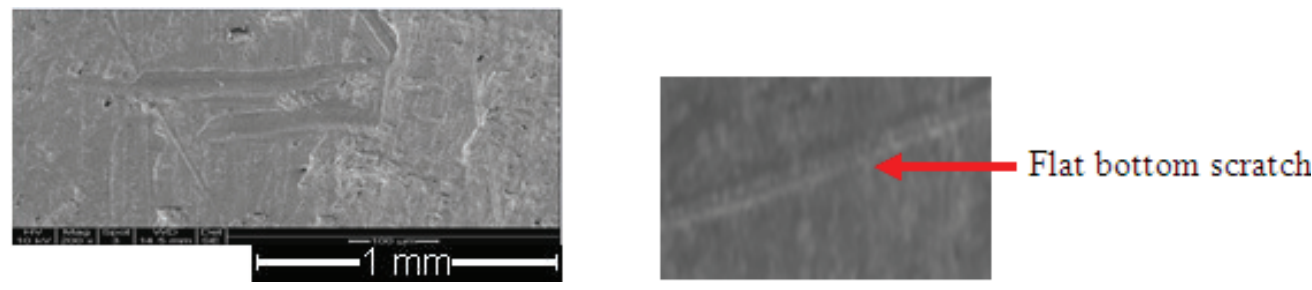

Fig. 1. ESEM images of the wire surface showing flat bottom scratches

The effects of the miniature scratches on the fracture behaviour of wires were investigated numerically using finite element simulations because the miniature defects are difficult to manufacture accurately experimentally. The finite element tensile testing simulations were conducted with the isotropic elastic-plastic model combined with the strain-based phenomenological shear failure criterion inbuilt in the Abaqus 6.9-1finite element (FE) code. The isotropic elastic-plastic model in Abaqus is based on a linear isotropic elasticity theory and a uniaxial-stress, plastic-strain strain-rate relationship [8]. The shear failure criterion is a phenomenological model for predicting the onset of damage due to shear band formation and localisation, and cracking within the shear bands [9]. Details of the isotropic elastic-plastic model and the modeling parameters; and the phenomenological shear failure model and the methodology for the determination of the phenomenological shear failure calibrated modeling parameters can be found in the work of [10]. 


\section{EXPERIMENTAL AND FE ANALYSIS PROCEDURES}

\subsection{LABORATORY TENSILE TESTING}

Two wire sizes with $12 \mathrm{~mm} \times 5 \mathrm{~mm}$ and $12 \mathrm{~mm} \times 7 \mathrm{~mm}$ cross-sectional dimensions were tested. Twenty four tensile specimens made from the as-received wires and twenty four tensile specimens with a $2 \mathrm{~mm} \times 2 \mathrm{~mm}$ (width and depth) channel shaped cut hereinafter referred to as the large notch shown in Figures 2(a) and (c) respectively were tested with an Instron IX4505 universal testing machine (UTM). The UTM was fitted with an Instron 2518 series load cell with a maximum static capacity of $\pm 100 \mathrm{kN}$ and the displacement was measured using an Instron 2630-112 clip-on strain gauge extensometer with a $50 \mathrm{~mm}$ gauge length. The dimension of the engineered channel-shaped cut was chosen to ensure that the engineered channel-shaped cut is substantially larger than any inherent channel-shaped scratch on the as-received wires in order to ensure that the engineered channel-shaped cut have a predominant effect on the tensile properties and fracture behaviour of the wires.

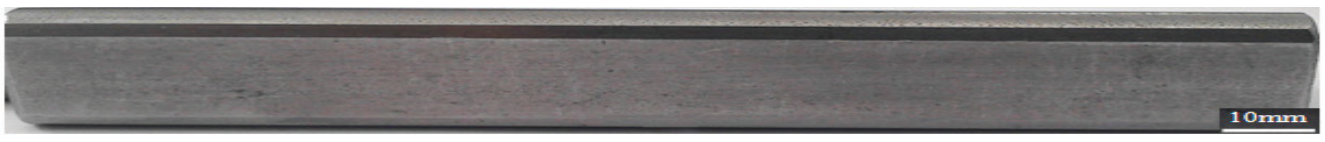

(a) Experimental wire specimen

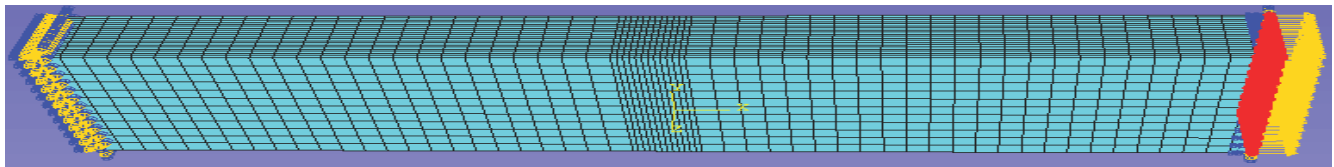

(b) FE model of wire specimen

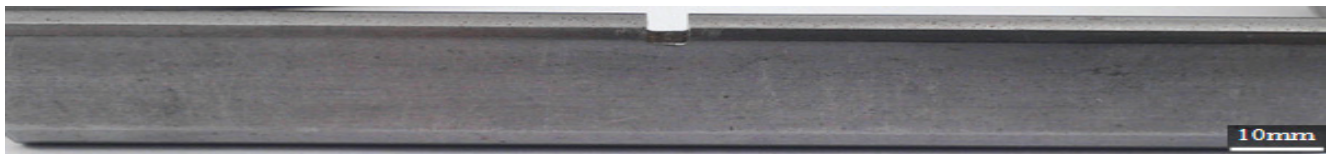

(c) Experimental wire specimen with a channel-shaped cut

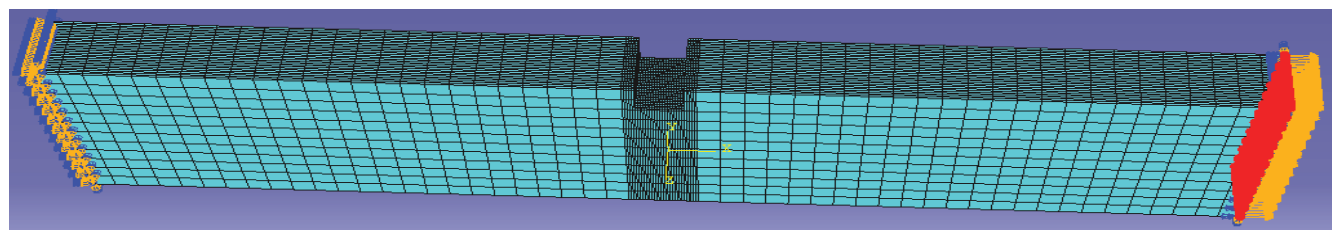

(d) FE model of wire specimen with a channel-shaped cut

Fig. 2. Laboratory and FE models of the wire specimen with and without a channel cut 


\subsection{Finite ELEMENT tensile testing SIMULATION.}

Three dimensional FE simulations of the tensile testing of the models of the as-received wire specimens and the wire specimens with a machined $2 \mathrm{~mm}$ x $2 \mathrm{~mm}$ channel-shaped cut shown in Figures 2(b) and (d) were conducted with the same material input data. The material input data employed for the simulation was that of the as-received wire specimen with tensile properties (yield load, ultimate load and displacement at fracture) closest to the mean of the twenty four samples tested. The simulation was conducted with the phenomenological shear damage and fracture model which has been established by [11] as an appropriate fracture model for the prediction of the fracture behaviour of the wires considered in this work.

The outer regions of the models of the as-received wire specimen and the wire specimen with the large notch were meshed with $1 \mathrm{mmx} 1 \mathrm{~mm} \times 1 \mathrm{~mm}$ C3D8R elements (8-node hexahedral linear brick reduced integration elements with hourglass control). The middle of the models of the as-received wire specimen and the wire specimen with the large notch were meshed with $0.0625 \mathrm{~mm}^{3}$ elements with an aspect ratio of 2:1 (that is based on the ratio of the width of the element in the direction of the thickness of the wire specimen to the thickness of the element in the direction of the length of the wire specimen) positioned with the length (longest dimension) in the direction of the width of the wire. These element size, aspect ratio and orientation have been found by [10] to be the appropriate element size, aspect ratio and orientation for accurate prediction of the fracture shape of the wire considered in this research. The FE tensile testing simulations were conducted by fixing the left hand ends of the models and subjecting the right hand ends of the models, which are free to move only in the direction of the tensile load (X-axis), to a longitudinal displacement as shown in Figures 2(b) and (d). The FE tensile testing simulation results for the wire specimens with and without the large notch were validated by comparing the FE and experimental force-displacement curves and by comparing the experimental and FE predicted fracture shapes. The validated FE simulation procedure was subsequently used to investigate the effects of the miniature across-the-thickness and across-the-width channel-shaped scratches with width and thickness from 0.1 to 0.19 (i.e. less than $0.2 \mathrm{~mm}$ ).

\section{RESULTS}

For both wire sizes, the variation of the test results between the different samples of the as-received wire specimens and the wire specimens with the large notch was small ( $\sim$ a few percent) and only the tensile test results of the $12 \mathrm{~mm} \times 7 \mathrm{~mm}$ wire specimens are presented. The normalised experimental force-displacement curves presented in Figures 3 and 4 are the curves of the as-received wire specimen and the wire specimen with the large notch, with tensile properties closest to the mean of the twenty four specimens tested. The FE predicted fractured sequences for the as-received 
$12 \mathrm{~mm} \times 7 \mathrm{~mm}$ wire specimen and for the $12 \mathrm{~mm} \times 7 \mathrm{~mm}$ wire specimen with the large notch are shown in Figures 5(a) to (e) and 6(a) to (e) respectively. The fracture process in the FE model is indicated by element(s) removal. Where the elements removed during the fracture initiation and/or fracture propagation are not visible in the meshed image, the wireframe images are presented to illustrate the fracture process sequences and paths. The fractured experimental specimen of the as-received $12 \mathrm{~mm} \times 7 \mathrm{~mm}$ wire specimen and that of the $12 \mathrm{~mm} \times 7 \mathrm{~mm}$ wire specimen with large notch are shown in Figures 5(d) and 6(d) respectively. The FE predicted fractured sequence for the $12 \mathrm{~mm}$ x $7 \mathrm{~mm}$ wire specimen with $0.19 \mathrm{~mm} \times 0.19 \mathrm{~mm}$ across-the-thickness scratches shown in Figure 7(a) and across-the-width scratches shown in Figure 8(a) are shown in Figures

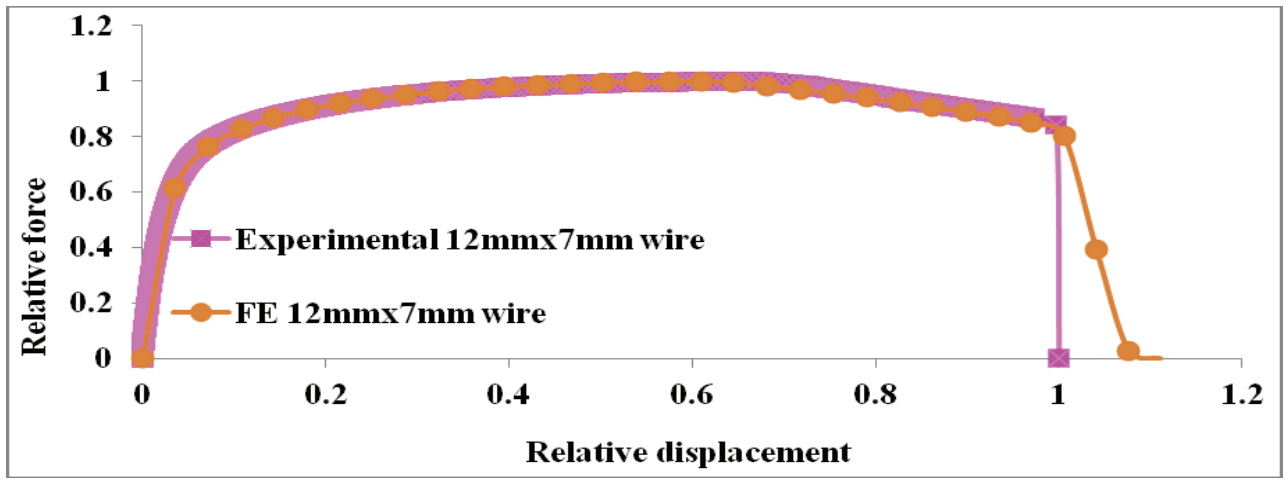

Fig. 3. Experimental and FE force-displacement curves for an as-received wire

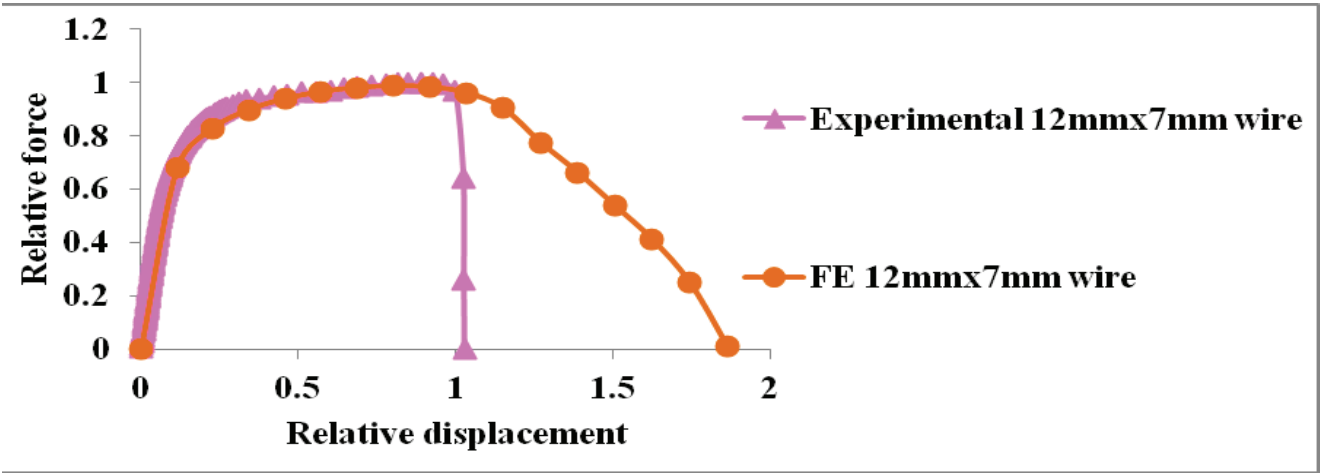

Fig. 4. Experimental and FE force-displacement curves for wires with a channel-shaped cut 
7(b) to (f) and Figures 8(b) to (f) respectively. Similar results in terms of the force-displacement curves, fracture sequences and fracture shapes were obtained from the experimental and FE tensile testing of the as-received $12 \mathrm{~mm} \times 5 \mathrm{~mm}$ wire specimens, the $12 \mathrm{~mm} \times 5 \mathrm{~mm}$ wire specimens with the large notch, and the $12 \mathrm{~mm} \times 5 \mathrm{~mm}$ wire specimens with the $0.19 \mathrm{~mm} \times 0.19 \mathrm{~mm}$ miniature across-the-thickness and across-the-width channel shaped scratches.

\section{Discussion}

The ability of the FE simulation to predict force-displacement curves, and cup and cone fracture shapes that agree with the experimental force-displacement curves and the cup and cone fracture exihibited by the as-received wire and the wire specimen with the large notch shown in Figures 5(d) and (e), and 6(e) and (f) respectively validates the FE simulation procedures' ability to predict the tensile and fracture behaviours of the wires with or without channel-shaped scratches/notches. In the as-received wire specimen, the wire specimen with the large notch, the wire specimen with the miniature across-thethickness scratch and the wire specimen with the miniature across-the-width scratch, fracture initiation occured at the locations of the maximum equivalent plastic strain indicated by the deepest red colour. The maximum equivalent plastic strain occurred at the center of the necked region of the as-received wire specimen, at the tip of the large notch, at both outer regions in the specimen with the miniature across-the-thickness scrath and at the tip of the across-the-width scratch as shown in Figures 5(a), 6(a), 7(b) and 8(b) respectively. Consequently, fracture initiation started at: the center of the necked region of the as-received wire specimen, at the tip of the large notch, at both outer regions in the specimen with the miniature across-the-thickness scratch and at the tip of the across-the-width scratch as shown in Figures 5(a), 6(a), 7(b) and 8(b) respectively.

The maximum equivalent plastic strain occurred at the center of the necked region of the as-received wire specimen, at the tip of the large notch and at the tip of the acrossthe-width scratch as expected because the strain concentration effects of the necked region, the large notch and the miniature across-the-width notch are the highest in the whole wire specimens. On the contrary, the maximum equivalent plastic strain did not occur at the tip of the miniature across-the-thickness scratch as expected but rather at the outer regions of the specimen with the miniature across-the-thickness scratch. This is because the strain concentration effect of the miniature across-the-thickness scratch with 0.02 relative depth (scratch depth to wire's thickness) compared with the strain concentration effect produced by the miniature across-the-width scratch with the same width and depth but with a 0.03 relative depth (scratch depth to wire's width) is not sufficient to make the amplified strain at the tip of the miniature across-the-thickness scratch the highest in the whole wire specimen. 
The fracture propagation paths in the as-received wire specimen, and the wire specimens with the large notch, the across-the-thickness scratch and the across-thewidth scratch are defined by the maximum equivalent plastic strain trajectory. The plastic strain trajectory is defined by the changing locations of the maximum equivalent plastic strain in the specimens with the applied longitudinal displacement(s) from the fracture initiation point to the end of the fracture process). Thus, following the maximum equivalent plastic strain trajectory, the initiated flat fracture at the center of the as-received wire specimen propagated vertically, transited to slant fracture, which propagated as shown in Figures 5(b) to (d), leading to the cup and cone fracture exhibited by the fractured model of the as-received wire specimen shown in Figure 5(e). Similarly, following the maximum equivalent plastic strain trajectory, the initiated slant fracture at the tip of the large notch propagated, transited to flat fracture, thereby forming a cup and cone fracture at the tip of the large notch as shown in Figures 6(b) to (c). The cup and cone fracture formed at the tip of the large notch propagated through the thickness of the wire as shown in Figures 6(d), leading to a cup and cone fracture exhibited by the wire specimen with the large notch shown in Figure 6(e).

Equally, following the maximum equivalent plastic strain trajectory, the propagation of the slant fracture initiated at both outer regions of the wire with the miniature across-the-thickness scrath toward the center of the wire occurred simultaneously with a flat fracture initiation at the center of the wire as shown in Figure $7 \mathrm{~b}$. This was followed by the simultaneous propagation of the slant and flat fractures vertically upward and downward as shown in Figure 7(c), leaving the wire with the miniature across-the-thickness scratch with a cup and cone fracture shape shown in Figure 7(d). It is essential to note that traces of the miniature across-the-thickness scrath can still be found at the location of the cup and cone fracture. This FE prediction agrees with what was observed experimentally by Smith and Easterling, (1993) who reported the presence of scratches at the location of fractured wires which exhibited a cup and cone fracture. 

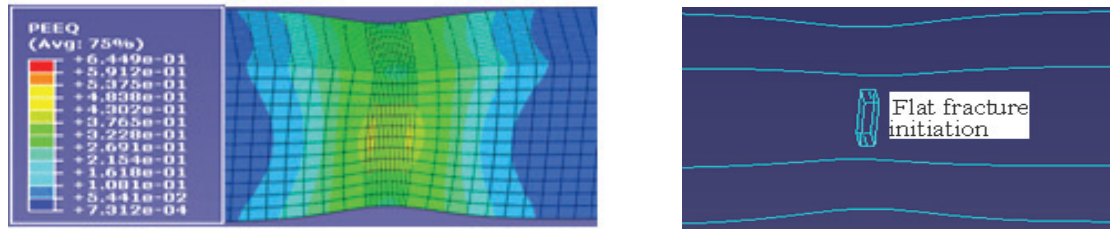

(a) Flat fracture initiation at the center of an as-received wire specimen
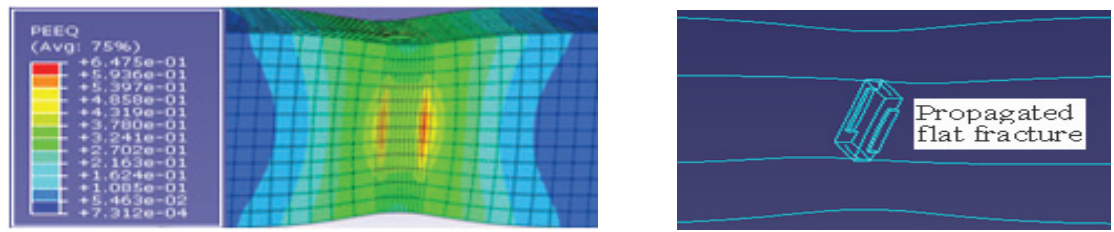

(b) Propagated flat fracture at the center of an as-received wire specimen
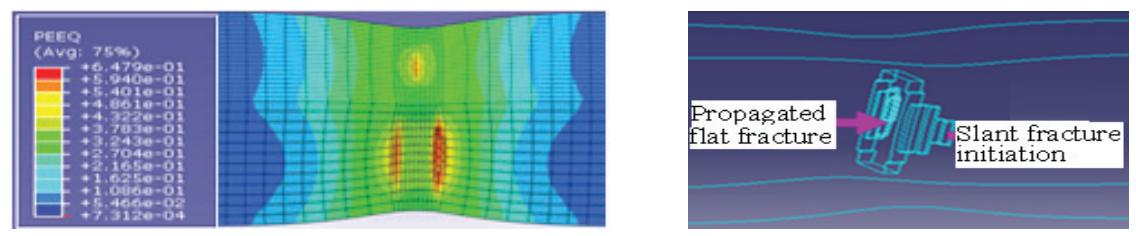

(c) Transition from flat to slant fracture at the center of the as-received wire specimen
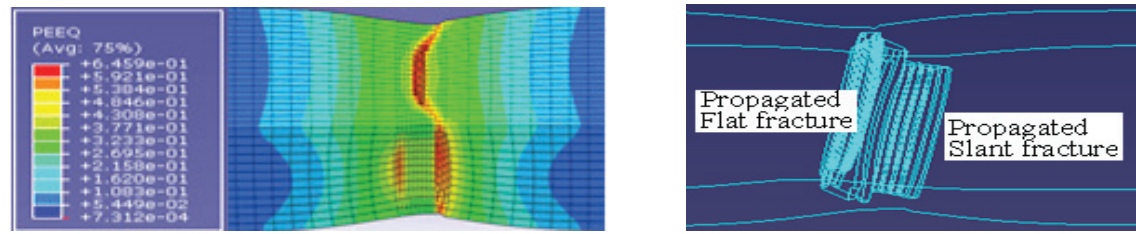

(d) Propagated flat and slant fractures at the center of the as-received wire specimen

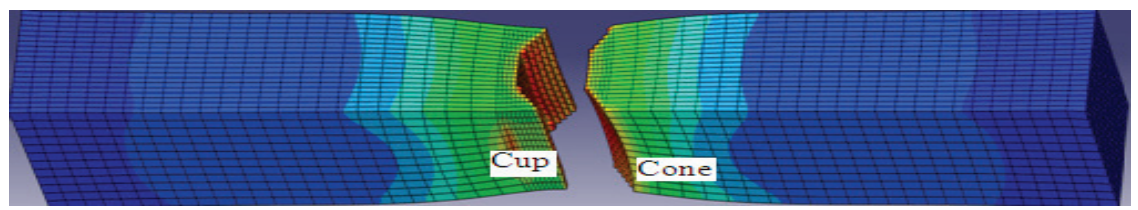

(e) Completely fractured as-received wire specimen
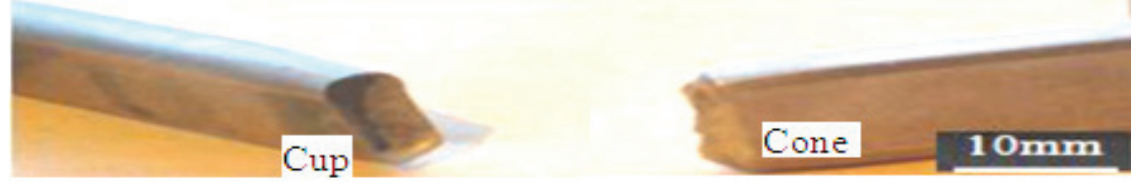

(f) Fractured experimental as-received wire specimen

Fig. 5. Fracture process sequence in an as-received wire specimen 

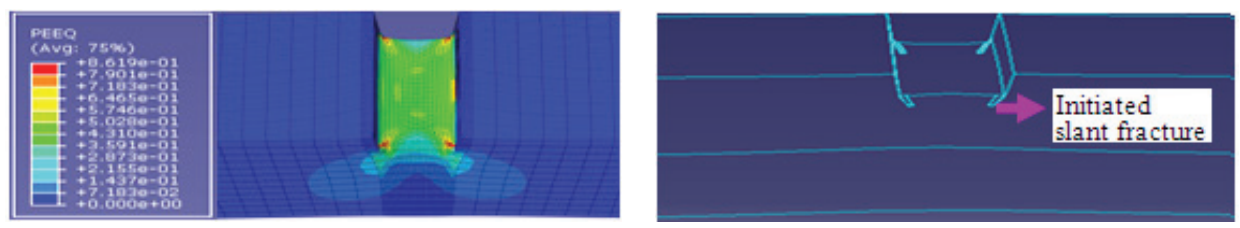

(a) Slant fracture initiation at notch root
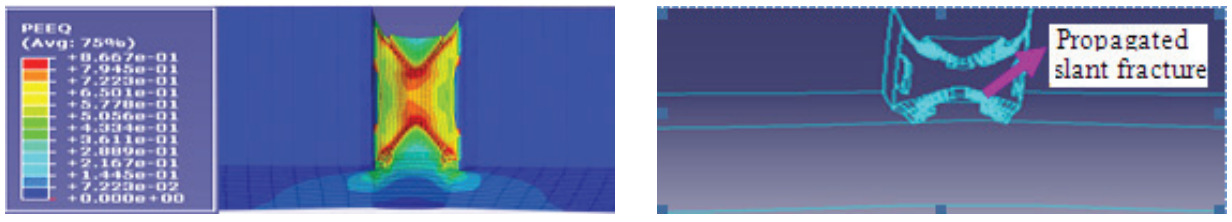

(b) Propagated slant fracture and initiated flat fracture
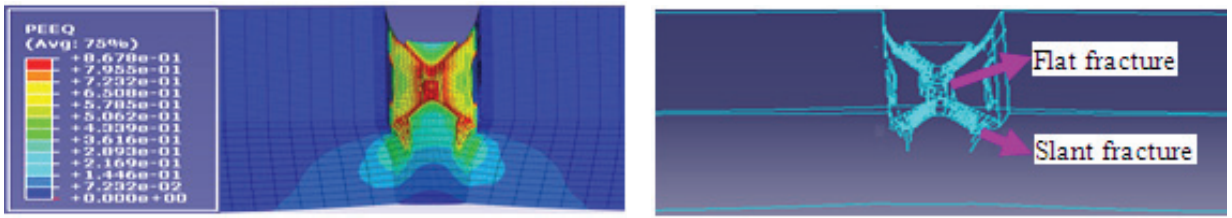

(c) Slant to flat fracture transition forming cup and cone at the top of wire specimen

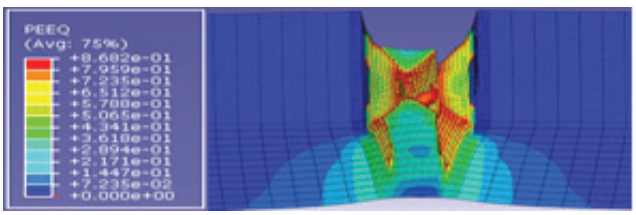

(d) Simultaneous flat and slant fracture (cup and cone fracture) propagation

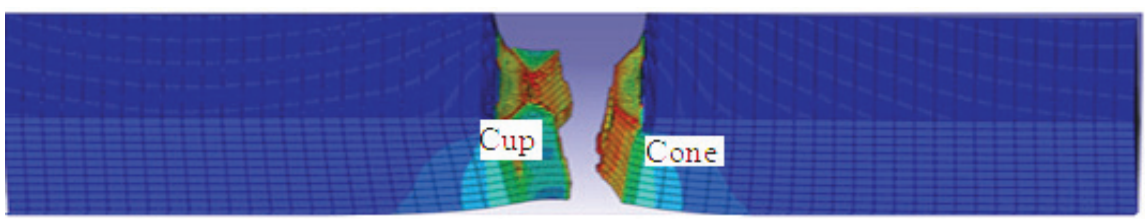

(e) Completely fractured specimen with cup and cone fracture

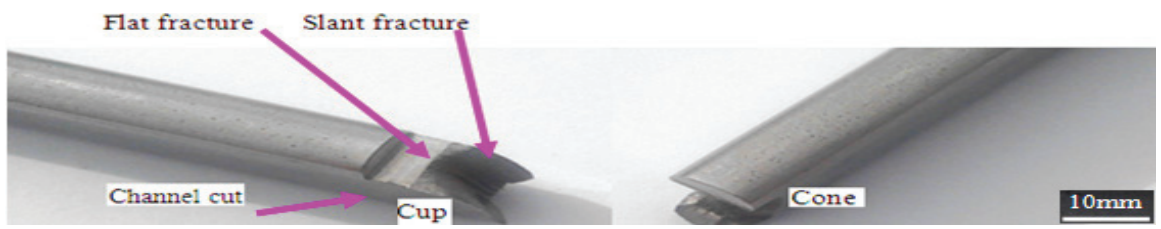

(f) Fractured experimental specimen of the wire with channel-shaped notch.

Fig. 6. Fracture process sequence in a wire specimen with a channel-shaped notch 

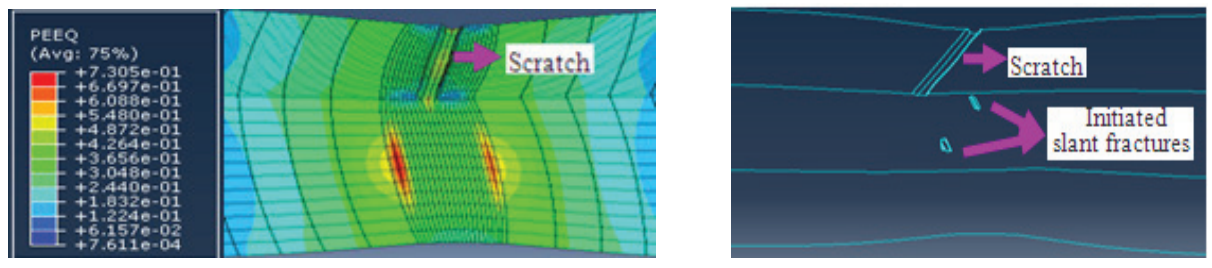

(a) Slant fracture initiation at the middle of the outer regions of the specimen
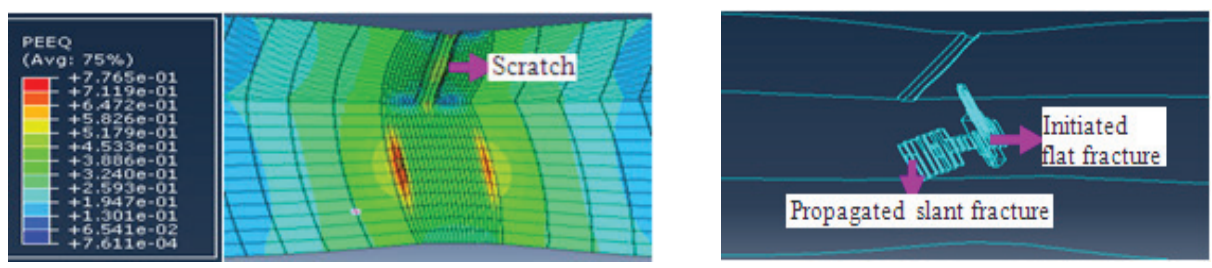

(b) Propagated slant fracture and initiated flat fracture at the middle of the wire specimen
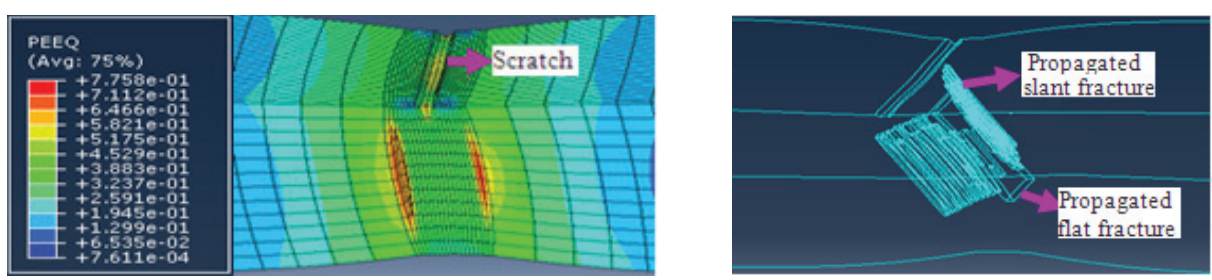

(c) Simultaneously propagated flat and slant fractures

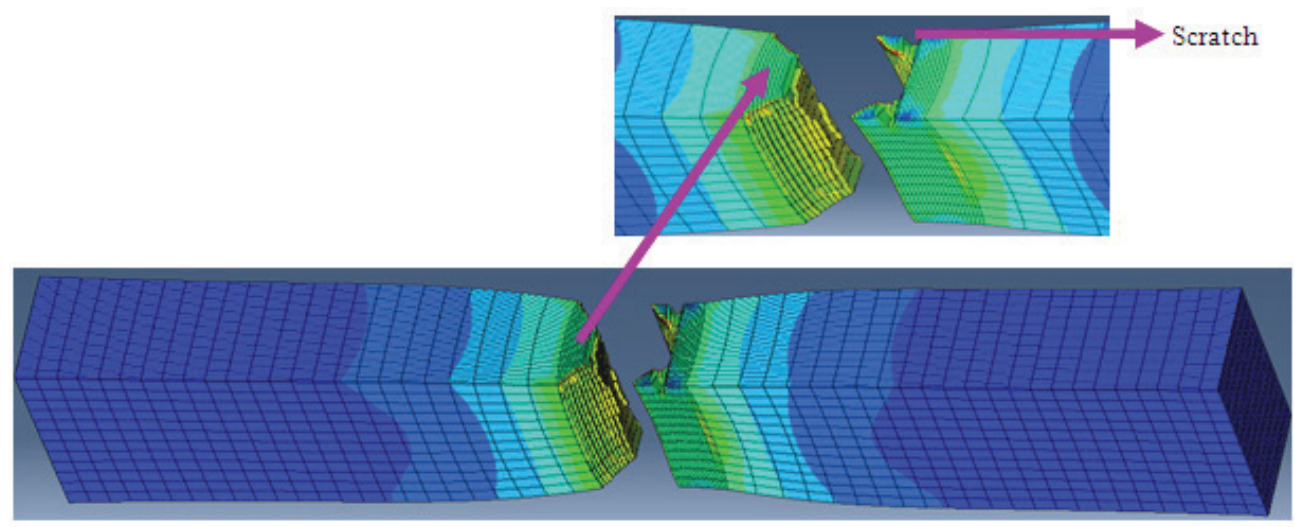

(d) Completely fractured specimen

Fig. 7. Predicted fracture sequence in wire specimen with across-the-thickness scratch 

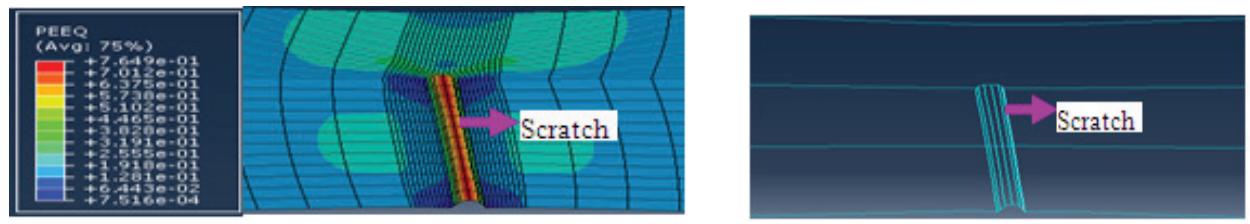

(a) Across-the-width scratch before fracture initiation
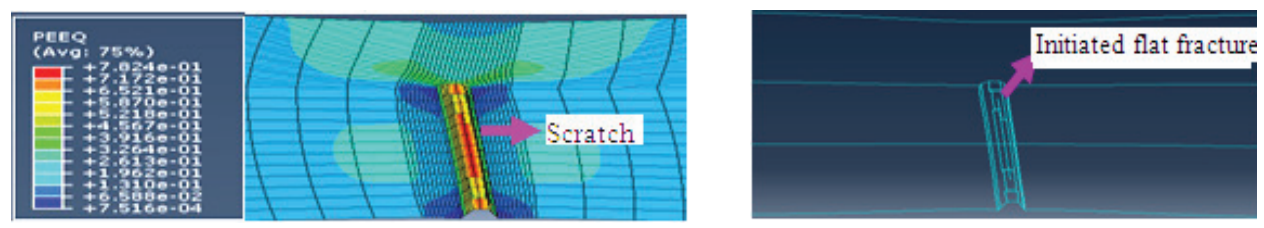

(b) Flat fracture initiation at the tip of the across-the-width scratch
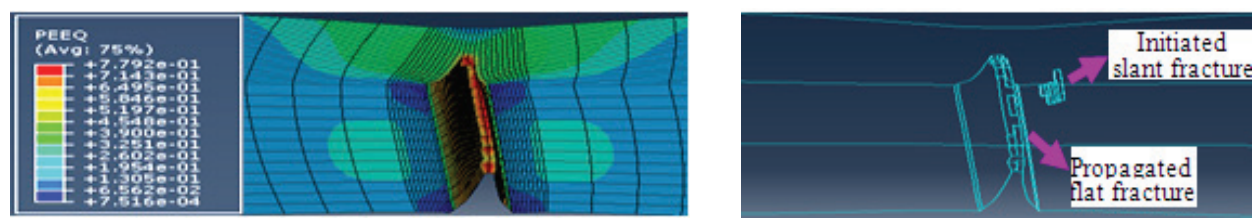

(c) Propagated flat fracture and initiated slant fracture
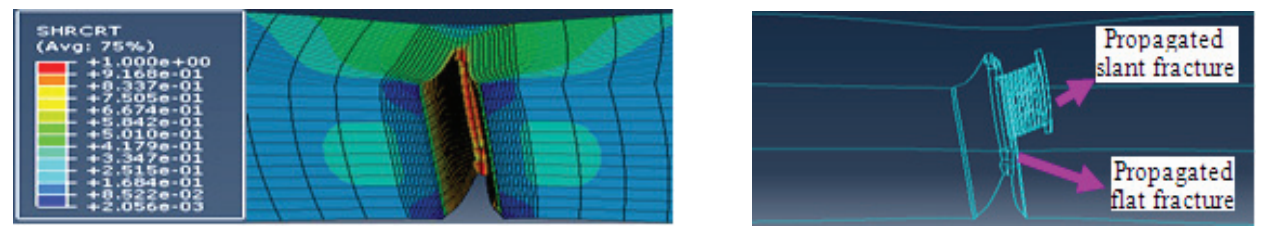

(d) Propagated slant fracture joining up with propagated flat fracture
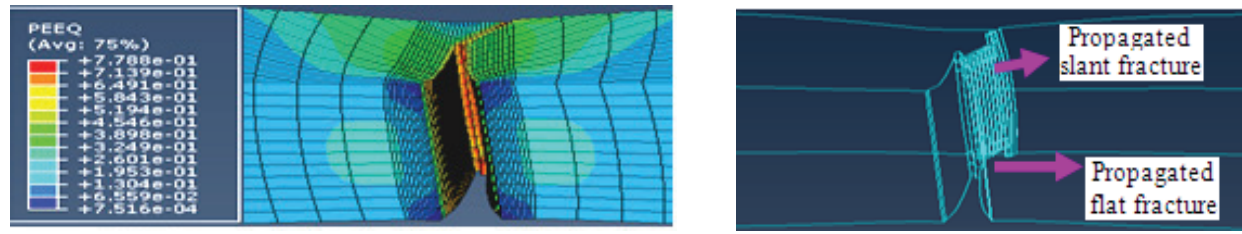

(e) Completely propagated flat and slant fracture

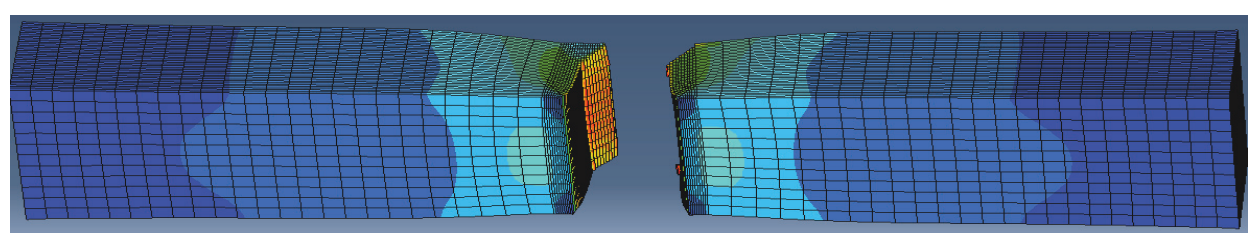

(f) Completely fractured model

Fig. 8. Predicted fracture sequence in a wire specimen with an across-the-width scratch 
The fracture propagation in the wire specimen with the miniature across-the-width scratch equally followed the maximum equivalent plastic strain trajectory, as the initiated flat fracture at the tip of the miniature across-the-width scratch propagated through approximately two thirds of the width of the wire as shown in Figure 8(c). This was followed by a slant fracture initiation at the middle of the remaining approximately one third of the wires' thickness, which propagated and liked up with the propagated flat fracture as shown in Figures 8(d). The slant fracture further propagated vertically through the width of the wire as shown in Figure 8(e), leaving the wire with the miniature across-the-width scratch with a flat and slant fracture shape shown in Figure 8(f).

\section{ConClusion}

In this paper, the FE simulation procedures employed for the prediction of the effects of miniature channel-shaped across-the-thickness and across-the-width scratches that are difficult to manufacture accurately experimentally are presented. This study revealed that the presence of both miniature channel-shaped across-the-thickness and acrossthe-width scratches changed the fracture behaviour of the wires in terms of the location of fracture initiation and the sequence of fracture propagation. The fracture initiation locations and the crack propagation paths in the wires are governed by the locations of the maximum equivalent plastic strain and the maximum equivalent plastic strain trajectory respectively. This study also revealed that the across-the-thickness scratch neither acted as a crack initiator because the amplified strain at the tip of the scratch was not the highest nor affected the fracture shape of the wire. Conversely, the acrossthe-width scartch acted as a crack initiator and changed the cup and cone fracture exhibited by the as-receievd wires to a fracture shape with a predominantly flat fracture. These results serve as a useful tool for understanding the fracture behaviour and failure analysis for typical wires used for civil engineering applications containing miniature channel-shaped scratches that are not detectable by the present inline electromagnetic defect detection system and whose effects on the fracture behaviour of the wires are difficult to investigate experimentally.

\section{REFERENCES}

1. Toribio J., And Ayaso F.J., 2003. Anisotropic fracture behaviour of cold drawn steel: a materials science approach. Materials Science and Engineering A343, Pages 265-272.

2. VANDER Voort G. F., 1987. Visual Examination and Light Microscopy. ASM Handbook, Volume 12: Fractography, Pages 91-165, ASM web. www.asminternational.org, Assessed $15^{\text {th }}$ July, 2013.

3. Sмith G.D.W., And Easterling K.E., 1993. Failure of Aircraft Target Towing Cables, Handbook of cases of histories in failure analysis, Vol 2, ASM International, ISBN: 0-87170-495-1.

4. Lothian E., Hermann K., Stewart M., 1981. An Atlas of Metal Damage. Wolf Publishing Limited, ISBN 0723407509. 
5. Toribio J., and Ayaso F. J., 2001. Fracture Performance of Progressively Drawn Pearlitic Steel under Triaxial Stress States. Materials Science, Volume 37, Number 5, Pages 707-717.

6. Toribio J., Valiente A., 2006. Failure analysis of cold drawn eutectoid steel wires for prestressed concrete, Engineering Failure Analysis, 13 (3), pages 301-311.

7. Fell Benjamin V., and Kanvinde Amit M., 2009. Recent fracture and fatigue research in steel structures, National Council of Structural Engineers Associations(NCSEA).http://www.structuremag.org/article. aspx?articleID=850, assessed 02/03/2012.

8. Simulia, 2007. Abaqus documentation, Abaqus Incorporated, Dassault Systemes.

9. Hooputra H., Gese H., Dell, H., and Werner H., 2004. A Comprehensive Failure Model for Crashworthiness Simulation of Aluminium Extrusions. International Journal of Crashworthiness, Vol. 9, no.5, Pages 449-464.

10. Adewole K. K., Bull S. J., 2013. Prediction of the fracture performance of defect-free steel bars for civil engineering applications using finite element simulation. Construction and Building Materials, Volume 41, pages 9-14.

11. Adewole K. K., 2013. Identification of Appropriate Micromechanical Fracture Model for Predicting Fracture Performance of Steel Wires for Civil Engineering Applications. Global Journal of Researches in Engineering, Civil And Structural Engineering. Volume 13 Issue Version 1.0, pages 25-34.

Received: 11.11.2013

Revised: 05.05.2014 